\title{
Color Image Enhancement by a Forward-and-Backward Adaptive Beltrami Flow
}

\author{
Nir A. Sochen ${ }^{1,2}$, Guy Gilboa ${ }^{1}$, and Yehoshua Y. Zeevi ${ }^{1}$ \\ 1 Department of Electrical Engineering, Technion - Israel Institute of Technology \\ Technion City, Haifa 32000, Israel \\ gilboa@tx.technion.ac.il \\ zeevi@ee.technion.ac.il \\ 2 Department of Applied Mathematics, University of Tel-Aviv \\ Ramat-Aviv, Tel-Aviv 69978, Israel \\ sochen@math.tau.ac.il
}

\begin{abstract}
The Beltrami diffusion-type process, reformulated for the purpose of image processing, is generalized to an adaptive forward-andbackward process and applied in localized image features' enhancement and denoising. Images are considered as manifolds, embedded in higher dimensional feature-spaces that incorporate image attributes and features such as edges, color, texture, orientation and convexity. To control and stabilize the process, a nonlinear structure tensor is incorporated. The structure tensor is locally adjusted according to a gradient-type measure. Whereas for smooth areas it assumes positive values, and thus the diffusion is forward, for edges (large gradients) it becomes negative and the diffusion switches to a backward (inverse) process. The resultant combined forward-and-backward process accomplishes both local denoising and feature enhancement.
\end{abstract}

Keywords: scale-space, image enhancement, color processing, Beltrami flow, anisotropic diffusion, inverse diffusion.

\section{Introduction}

Image denoising, enhancement and sharpening are important operations in the general fields of image processing and computer vision. The success of many applications, such as robotics, medical imaging and quality control depends in many cases on the results of these operations. Since images cannot be described as stationary processes, it is useful to consider local adaptive filters. These filters are best described as solutions of partial differential equations (PDE).

The application of PDE's in image processing and analysis starts with the linear scale-space approach $[23,8]$ which applies the heat equation by considering the noisy image as an initial condition. The associated filter is a Gaussian with a time varying scale. Perona and Malik [10] in their seminal contribution, generalized the heat equation to a non-linear diffusion equation where the diffusion coefficient depends upon image features i.e. edges. This work paved the 
way for a variety of PDE based methods that were applied to various problems in low-level vision (see [17] for an excellent introduction and overview).

The Beltrami framework was recently proposed by Sochen et al [13] as a viewpoint that unifies many different algorithms and offer new possibilities of definitions and solutions of various tasks. Images and other vision objects of interest such as derivatives, orientations, texture, sequence of images, disparity in stereo vision, optical flow and more, are described as embedded manifolds. The embedded manifold is equipped with a Riemannian structure i.e. a metric. The metric encodes the geometry of the manifold. Non-linear operations on these objects are done according to the local geometry of the specific object of interest. The iterative process is understood as an evolution of the manifold. The evolution is a consequence of a non-linear PDE. No global (timewise) kernels can be associated with these non-linear PDE's. Short time kernels for these processes were derived recently in [15].

We generalize the works of Perona and Malik [10], Sochen et al [13] and Weickert [18] and show how one can design a structure tensor that controls the non-linear diffusion process starting from the induced metric that is given in the Beltrami framework. The proposed structure tensor is non-definite positive or negative and switches between them according to image features. This results in a forward-and-backward diffusion flow. Different regions of the image are forward or backwards diffused according to the local geometry within a neighborhood. The adaptive property of the process, that expresses itself in the local decision on the direction of the diffusion and on its strength, is the main novelty of this paper.

\section{A Geometric Measure on Embedded Maps}

\subsection{Images as Riemannian Manifolds}

According to the geometric approach to image representation, images are considered to be two-dimensional Riemannian surfaces embedded in higher dimensional spatial-feature Riemannian manifolds $[13,5,6,5,7,16,14]$. Let $\sigma^{\mu}, \quad \mu=1,2$, be the local coordinates on the image surface and let $X^{i}, \quad i=1,2, \ldots, m$, be the coordinates of the embedding space than the embedding map is given by

$$
\left(X^{1}\left(\sigma^{1}, \sigma^{2}\right), X^{2}\left(\sigma^{1}, \sigma^{2}\right), \ldots, X^{m}\left(\sigma^{1}, \sigma^{2}\right)\right) .
$$

Riemannian manifolds are manifolds endowed with a bi-linear positivedefinite symmetric tensor which constitutes a metric. Denote by $\left(\Sigma,\left(g_{\mu \nu}\right)\right)$ the image manifold and its metric and by $\left(M,\left(h_{i j}\right)\right)$ the spatial-feature manifold and its corresponding metric. The induced metric can be calculated by $g_{\mu \nu}=$ $h_{i j} \partial_{\mu} X^{i} \partial_{\nu} X^{j}$. The map X : $\Sigma \rightarrow M$ has the following weight [11]

$$
E\left[X^{i}, g_{\mu \nu}, h_{i j}\right]=\int d^{2} \sigma \sqrt{g} g^{\mu \nu}\left(\partial_{\mu} X^{i}\right)\left(\partial_{\nu} X^{j}\right) h_{i j}(\mathbf{X})
$$

where the range of indices is $\mu, \nu=1,2$, and $i, j=1, \ldots, m=\operatorname{dim} M$, and we use the Einstein summation convention: identical indices that appear one up and 
one down are summed over. We denote by $g$ the determinant of $\left(g_{\mu \nu}\right)$ and by $\left(g^{\mu \nu}\right)$ the inverse of $\left(g_{\mu \nu}\right)$. In the above expression $d^{2} \sigma \sqrt{g}$ is an area element of the image manifold. The rest, i.e. $g^{\mu \nu}\left(\partial_{\mu} X^{i}\right)\left(\partial_{\nu} X^{j}\right) h_{i j}(\mathbf{X})$, is a generalization of $L_{2}$. It is important to note that this expression (as well as the area element) does not depend on the choice of local coordinates.

The feature evolves in a geometric way via the gradient descent equations

$$
X_{t}^{i} \equiv \frac{\partial X^{i}}{\partial t}=-\frac{1}{2 \sqrt{g}} h^{i l} \frac{\delta E}{\delta X^{l}} .
$$

Note that we used our freedom to multiply the Euler-Lagrange equations by a strictly positive function and a positive definite matrix. This factor is the simplest one that does not change the minimization solution while giving a reparameterization invariant expression. This choice guarantees that the flow is geometric and does not depend on the parameterization.

Given that the embedding space is Euclidean, the variational derivative of $E$ with respect to the coordinate functions is given by

$$
-\frac{1}{2 \sqrt{g}} h^{i l} \frac{\delta E}{\delta X^{l}}=\Delta_{g} X^{i}=\frac{1}{\sqrt{g}} \partial_{\mu}\left(\sqrt{g} g^{\mu \nu} \partial_{\nu} X^{i}\right),
$$

where the operator that is acting on $X^{i}$ in the first term is the natural generalization of the Laplacian from flat surfaces to manifolds. In terms of the formalism implemented in our study, this is called the second order differential parameter of Beltrami [9], or in short Beltrami operator.

\subsection{The Metric as a Structure Tensor}

There has been a few works using anisotropic diffusion processes. Cottet and Germain [2] used a smoothed version of the image to direct the diffusion, while Weickert $[20,19]$ smoothed also the structure tensor $\nabla I \nabla I^{T}$ and then manipulated its eigenvalues to steer the smoothing direction. Eliminating one eigenvalue from a structure tensor, first proposed as a color tensor in [3], was used in [12], in which the tensors are not necessarily positive definite. While in $[21,22]$, the eigenvalues are manipulated to result in a positive definite tensor. See also [1], where the diffusion is in the direction perpendicular to the maximal gradient of the three color channels (this direction is different than that of [12]).

Let us first show that the diffusion directions can be deduced from the smoothed metric coefficients $g_{\mu \nu}$ and may thus be included within the Beltrami framework under the right choice of directional diffusion coefficients.

The induced metric $\left(g_{\mu \nu}\right)$ is a symmetric uniformly positive definite matrix that captures the geometry of the image surface. Let $\lambda_{1}$ and $\lambda_{2}$ be the largest and the smallest eigenvalues of $\left(g_{\mu \nu}\right)$, respectively. Since $\left(g_{\mu \nu}\right)$ is a symmetric positive matrix its corresponding eigenvectors $u_{1}$ and $u_{2}$ can be chosen orthonormal. Let $U \equiv\left(u_{1} \mid u_{2}\right)$, and $\Lambda \equiv\left(\begin{array}{cc}\lambda_{1} & 0 \\ 0 & \lambda_{2}\end{array}\right)$, then we readily have the equality

$$
\left(g_{\mu \nu}\right)=U \Lambda U^{T} .
$$


Note also that

$$
\left(g^{\mu \nu}\right) \equiv\left(g_{\mu \nu}\right)^{-1}=U \Lambda^{-1} U^{T}=U\left(\begin{array}{cc}
1 / \lambda_{1} & 0 \\
0 & 1 / \lambda_{2}
\end{array}\right) U^{T},
$$

and that

$$
g \equiv \operatorname{det}\left(g_{\mu \nu}\right)=\lambda_{1} \lambda_{2}
$$

Our proposed enhancement procedure will control those eigenvalues adaptively so that only meaningful edges will be enhanced, where smooth areas will be denoised.

\section{New Adaptive Structure Tensor}

\subsection{Changing the Eigenvalues}

From the above derivation of the metric $g_{\mu \nu}$, it follows that the larger eigenvalue $\lambda_{1}$ corresponds to the eigenvector in the gradient direction (in the 3D Euclidean case: $\left.\left(I_{x}, I_{y}\right)\right)$. The smaller eigenvalue $\lambda_{2}$ corresponds to the eigenvector perpendicular to the gradient direction (in the 3D Euclidean case: $\left(-I_{y}, I_{x}\right)$ ). The eigenvectors are equal for both $g_{\mu \nu}$ and its inverse $g^{\mu \nu}$, whereas the eigenvalues have reciprocal values. We can use the eigenvalues as a means to control the Beltrami flow process. For convenience let us define $\lambda^{1} \equiv \frac{1}{\lambda_{1}}$. As the first eigenvalue of $g^{\mu \nu}$ (that is $\lambda^{1}$ ) increases, so does the diffusion force in the gradient direction. Thus, by changing this eigenvalue we can reduce, eliminate or even reverse the diffusion process across the gradient.

What would be the best strategy to control the diffusion process via adjustment of the relevant parameters ? There are a few requirements that might be considered as guidelines :

- The enhancement should essentially be with relevance to the important features, while originally smooth segments should not be enhanced.

- The contradictory processes of enhancement and noise reduction by smoothing (filtering) should coexist.

- The process should be as stable as possible, though restoration and enhancement processes are inherently unstable.

Let us define $\hat{\lambda}^{1}(s)$ as a new adaptive eigenvalue to be put instead of the original $\lambda^{1}$. We propose that this new eigenvalue will be proportional to the combined gradient magnitude of the three channels (colors) $\left|\nabla I_{\Sigma}\right|$ (that is $\hat{\lambda}^{1}=$ $\hat{\lambda^{1}}\left(\left|\nabla I_{\Sigma}\right|\right)$ in the following way:

$$
\hat{\lambda^{1}}(s)= \begin{cases}1-\left(s / k_{f}\right)^{n} & , 0 \leq s \leq k_{f} \\ \alpha\left[\left(\left(s-k_{b}\right) / w\right)^{2 m}-1\right] & , k_{b}-w \leq s \leq k_{b}+w \\ 0 & , \text { otherwise }\end{cases}
$$

and its smoothed version: 


$$
{\hat{\lambda^{1}}}_{\sigma}(s)=\hat{\lambda^{1}}(s) \star G_{\sigma}(s)
$$

where $\left|\nabla I_{\Sigma}\right| \equiv\left(\Sigma_{i}\left|\nabla I_{i}\right|^{2}\right)^{1 / 2}, \star$ denotes convolution, and $k_{f}<k_{b}-w$. We chose the exponent parameters $n$ and $m$ to be 4 and 1, respectively.

The new structure tensor has to be continuous and differentiable. In the discrete domain, (8) could suffice (although it is only piecewise differentiable), whereas (9) can fit the general continuous case. Other types of eigenvalue manipulation with similar nature may be considered.

The parameter $k_{f}$ is essentially the limit of gradients to be smoothed out, whereas $k_{b}$ and $w$ define the range of the backward diffusion, and should assume values of gradients that we want to emphasize. In our formula the range is symmetric, and we restrain the width from overlapping the forward diffusion area. One way of choosing these parameters in the discrete case, is by calculating the mean absolute gradient (MAG).

The parameter $\alpha$ determines the ratio between the backward and forward diffusion. Under the condition of $\alpha$ that renders the backward diffusion process to become too dominant, the stabilizing forward process can no longer avoid oscillations. One can avoid the evolution of new singularities in smooth areas by bounding the maximum flux resulting from the backward diffusion to be smaller than the maximum affected by the forward one. Formally, we say:

$$
\max _{s<k_{f}}\{s \lambda(s)\}>\max _{k_{b}-w<s<k_{b}+w}\{s \lambda(s)\}
$$

In the case of our proposed eigenvalue, we get a simple formula for $\alpha$, which just obeys this inequality by:

$$
\alpha=k_{f} / 2 k_{b} \quad \text {,for any } 0<w<k_{b}-k_{f}
$$

In practical applications, this bound can be doubled in value without experiencing major instabilities.

See [4] for elaboration on the forward and backward diffusion for signal enhancement.

\subsection{The Algorithm}

The algorithm to implement the flow $\mathbf{I}_{t}=\Delta_{\hat{g}} \mathbf{I}$ for color image enhancement is as follows:

1. Compute the metric coefficients $g_{\mu \nu}$. For the $N$ channel case (for color $N=3$ ) we have

$$
g_{\mu \nu}=\delta_{\mu \nu}+\sum_{k=1}^{N} I_{\mu}^{k} I_{\nu}^{k} .
$$


2. Diffuse the $g_{\mu \nu}$ coefficients by convolving with a Gaussian of variance $\rho$, thereby

$$
\tilde{g}_{\mu \nu}=G_{\rho} * g_{\mu \nu} .
$$

For $2 D$ images $G_{\rho}=e^{-\left(x^{2}+y^{2}\right) / \rho^{2}}$.

3. Compute the inverse smoothed metric $\tilde{g}^{\mu \nu}$. Change the eigenvalues of the inverse metric $\lambda^{1}, \lambda^{2},\left(\lambda^{1}<\lambda^{2}\right)$, of $\left(\tilde{g}^{\mu \nu}\right)$ so that $\lambda^{1}=\hat{\lambda}^{1}(s)$ and $\lambda_{2}=a$, $(a \geq 1)$. This yields a new inverse structure tensor $\hat{g}_{\mu \nu}$ that is given by:

$$
\left(\hat{g}_{\mu \nu}\right)=\tilde{U}\left(\begin{array}{cc}
\hat{\lambda}^{1}(s) & 0 \\
0 & a
\end{array}\right) \tilde{U}^{T}=\tilde{U} \hat{\Lambda} \tilde{U}^{T} .
$$

4. Calculate the determinant of the new structure tensor. Note that $\hat{g}$ can now

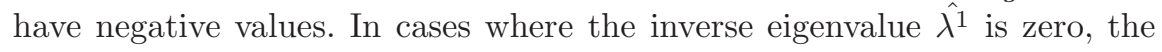
structure tensor determinant should assume a large value $M>>1$.

$\hat{g} \equiv \operatorname{det}\left(\hat{g}_{\mu \nu}\right)=\hat{\lambda_{1}} \hat{\lambda_{2}}=\frac{1}{\hat{\lambda^{1}}{\hat{\lambda^{2}}}^{2}}$,

$$
\hat{g}= \begin{cases}1 / a \lambda^{1} \hat{(}(s) & , \hat{\lambda^{1}}(s) \neq 0 \\ M & , \text { otherwise }\end{cases}
$$

5. Evolve the $k$-th channel via the Beltrami flow

$$
I_{t}^{k}=\Delta_{\hat{g}} I^{k} \equiv \frac{1}{\sqrt{\hat{g}}} \partial_{\mu}\left(\sqrt{\hat{g}} \hat{g}^{\mu \nu} \partial_{\nu} I^{k}\right)
$$

Remark: In this flow, we will not get imaginary values, though we have the term $\sqrt{\hat{g}}$ because in cases of negative $\hat{g}$ the constant imaginary term $i \equiv \sqrt{-1}$ will be canceled.

\subsection{Variations to the Scheme}

As the process involves inverse diffusion for enhancement - it is by definition not stable. To obtain a more stable process, which will denoise the image and preserve its edges, setting $\alpha=0$ will remove the inverse diffusion part, and leave us with a coherent denoising scheme.

There are a few ways to increase regularity in this PDE-based approach. One can replace the proposed conductance coefficient Eq. (8) by the smoothed one, Eq. (9). As presented in the algorithm, convolving the metric with a smoothing kernel, before manipulating it, increases the stability of the process. It is possible also to smooth smaller scales in a noisy signal by preprocessing. As we enhance the signal afterwards, this smoothing process does not affect the end result that much and enables us to operate in an originally much noisier environment. Finally, operating in extremely noisy areas, when we know of the type of singularity, we can apply more pre-smoothing, and consider only the largest gradient within the backward diffusion range.

We can substitute the dependency of $\hat{\lambda}^{1}$ instead of on the gradient, on similar "edge detectors": 


$$
\hat{\lambda}^{1}=\hat{\lambda}^{1}(g)
$$

or the smoothed version:

$$
\hat{\lambda^{1}}=\hat{\lambda}^{1}\left(G_{\rho} \star g\right)
$$

or on the original eigenvalue itself :

$$
\hat{\lambda^{1}}=\hat{\lambda}^{1}\left(\lambda^{1}\right)
$$

A local approach, that adjusts the parameters $k_{f}, k_{b}, w$ to be of different values in different segments of the image, is currently investigated.

\section{Results and Conclusion}

The image feature enhancement procedure developed in the framework of geometry, incorporates a nonlinear adaptive structure tensor that controls the enhancement process along gradients. In other words, the structure tensor is locally adjusted according to a gradient-type measure. Whereas for smooth areas it assumes positive values, and thus the diffusion is forward, for edges it becomes negative and the diffusion switches to a backward (inverse) process. In this way we accomplish both of the conflicting tasks of local denoising and feature enhancement.

In Figure 1 the left eye of the Mandrill image is shown, before and after the application of the adaptive Beltrami process. It depicts efficient denoising of the retina, with sharp edges somewhat enhanced. In Figure 2 a blurred and noisy Tulip photo is processed, enhancing the center of the flower while denoising its background. In a detail enlargement of the same image (the flower's pattern in Fig. 3) one can see more clearly that the bright curly outline of the leaf is enhanced (brighter in its center), whereas smooth areas are denoised.

[ For a closer look at the color images, please follow the web link: http://wwwvisl.technion.ac.il/belt-fab ] .

Lastly, note that the general scheme can be easily degenerated into a coherent stable denoising scheme that preserves edges.

\section{Acknowledgement}

This research has been supported in part by the Ollendorf Minerva Center, by the Fund for the Promotion of Research at the Technion, and by the Consortium for Broadband Communication administered by the Chief Scientist of the Israeli Ministry of Industrial and Commerce. 


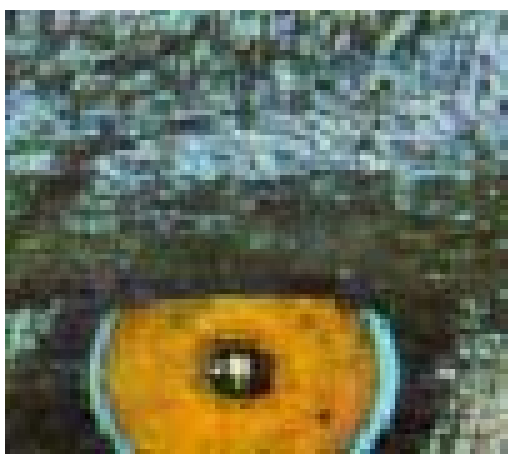

Original

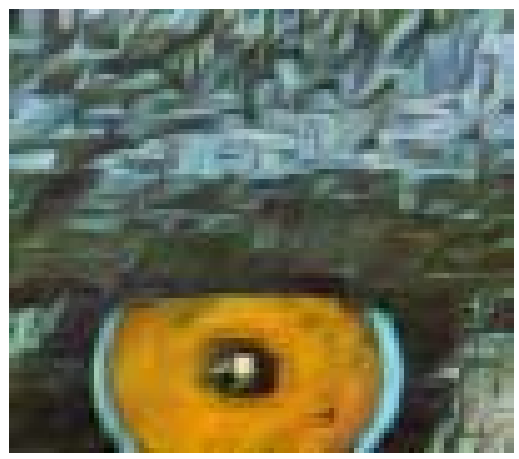

Beltrami-tensor-adaptive-local beta $=40$ ro $=1$ iter $=70$

Fig. 1. Left - original eye image, right - enhanced and denoised eye image. 70 iterations, $\left[k_{f}, k_{b}, w\right]=[0.5,4,2] * M A G, \rho=1$
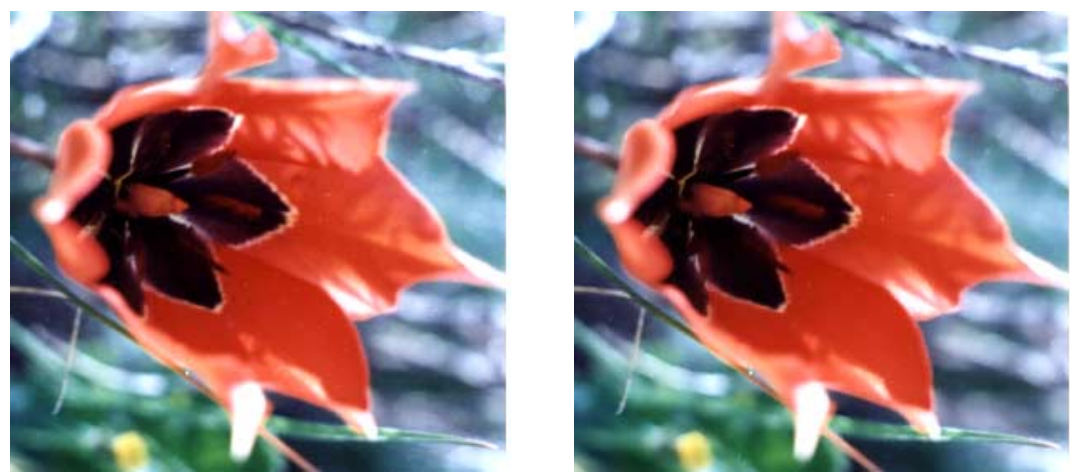

Fig. 2. Left - original tulip, right - enhanced and denoised tulip. 40 iterations, $\left[k_{f}, k_{b}, w\right]=[0.7,5,3] * M A G, \rho=1$

\section{References}

1. A. Chambolle, Partial Differential Equations and Image processing, Proceedings IEEE ICIP, 1 (1994) 16-20. 321

2. G. H. Cottet and L. Germain, Image processing through reaction combined with nonlinear diffusion, Math. Comp., 61 (1993) 659-673. 321

3. S. Di Zenzo, A note on the gradient of a multi image, Computer Vision, Graphics, and Image Processing, 33 (1986) 116-125. 321

4. G. Gilboa, N. Sochen, Y. Y. Zeevi, "Anisotropic selective inverse diffusion for signal enhancement in the presence of noise", to appear in IEEE ICASSP'2000, Istanbul, 2000. 323

5. R. Kimmel, N. Sochen and R. Malladi, "On the geometry of texture", Report, Berkeley Labs. UC, LBNL-39640, UC-405, November,1996. 320 

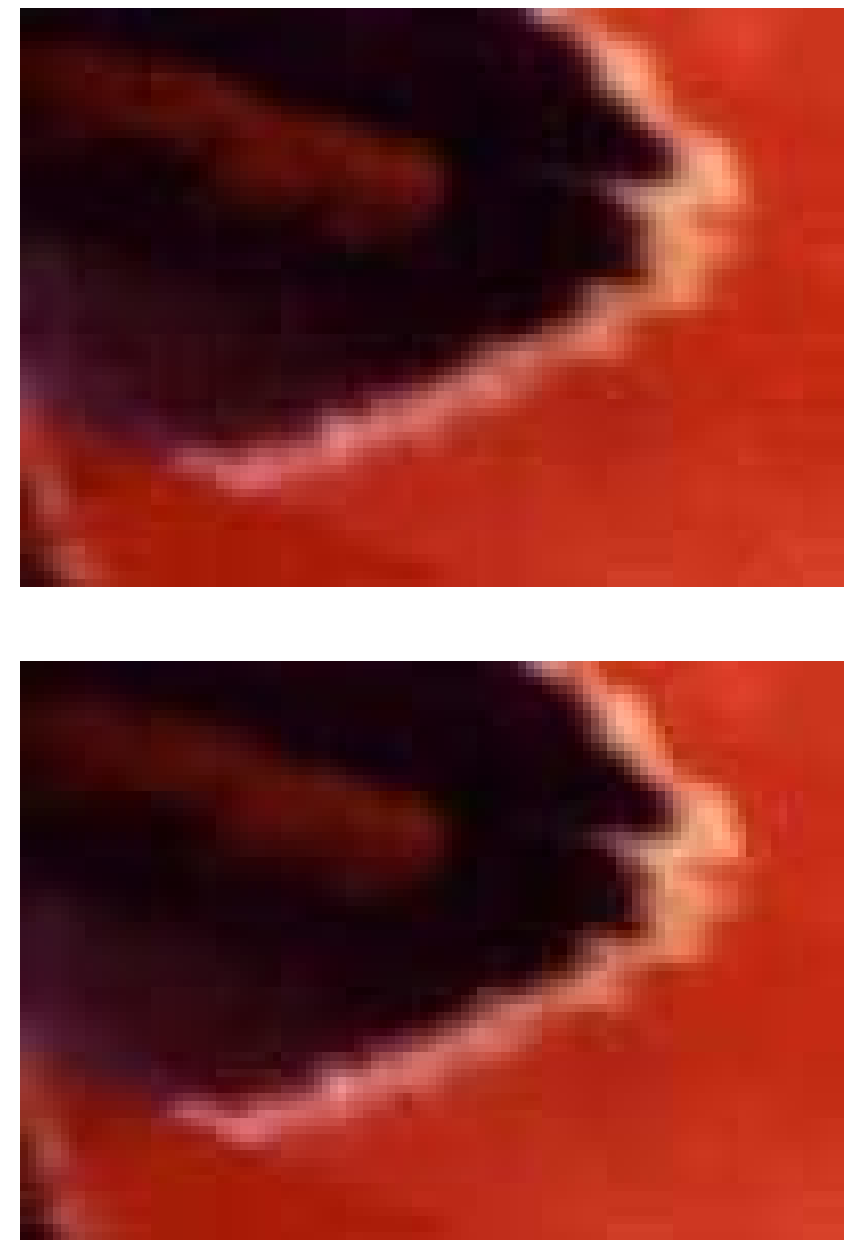

Fig. 3. Enlargement of Fig. 2 - a pattern on the tulip: top - original, bottom - enhanced and denoised image. Note the white ridge at the center of the leaf's outline.

6. R. Kimmel, R. Malladi and N. Sochen, "Images as Embedding Maps and Minimal Surfaces: Movies, Color, Texture, and Volumetric Medical Images", Proc. of IEEE CVPR'97, (1997) 350-355. 320

7. R. Kimmel, N. Sochen and R. Malladi, "From High Energy Physics to Low Level Vision", Lecture Notes In Computer Science: 1252, First International Conference on Scale-Space Theory in Computer Vision, Springer-Verlag, 1997, 236-247. 320

8. J. J. Koenderink, "The structure of images", Biol. Cybern., 50: 363-370, 1984. 319 
9. E. Kreyszing, "Differential Geometry", Dover Publications, Inc., New York, 1991. 321

10. P. Perona and J. Malik, "Scale-space and edge detection using anisotropic diffusion", IEEE Trans. Pat. Anal. Machine Intel., vol. PAMI-12,no. 7, pp. 629-639, 1990. 319, 320

11. A. M. Polyakov, "Quantum geometry of bosonic strings", Physics Letters, 103B (1981) 207-210. 320

12. G. Sapiro and D. L. Ringach, Anisotropic Diffusion of multivalued images with applications to color filtering, IEEE Trans. Image Proc., 5 (1996) 1582-1586. 321

13. N. Sochen, R. Kimmel and R. Malladi, "A general framework for low level vision", IEEE Trans. on Image Processing, 7, (1998) 310-318. 320

14. N. Sochen and Y. Y. Zeevi, "Images as manifolds embedded in a spatial-feature non-Euclidean space", November 1998, EE-Technion report no. 1181. 320

15. N. Sochen, "Stochastic processes in vision I: From Langevin to Beltrami", EETechnion technical report No. 245320

16. N. Sochen and Y. Y. Zeevi, "Representation of colored images by manifolds embedded in higher dimensional non-Euclidean space", IEEE ICIP'98, Chicago, 1998. 320

17. B. M. ter Haar Romeny Ed., Geometry Driven Diffusion in Computer Vision, Kluwer Academic Publishers, 1994. 320

18. J. Weickert, "Coherence-enhancing diffusion of colour images", Image and Vision Comp., 17 (1999) 199-210. 320

19. J. Weickert, "Multiscale texture enhancement", Computer analysis of images and patterns; Lecture Notes in Computer Science, 970 (1995), pp. 230-237, Springer. 321

20. J. Weickert, "Anisotropic diffusion in image processing", Ph.D. Thesis, Kaiserslautern University, Germany, November 1995321

21. J. Weickert, "Scale-space properties of nonlinear diffusion filtering with diffusion tensor", Report No. 110, Laboratory of Technomathematics, University of Kaiserslautern, 1994. 321

22. J. Weickert, Coherence-enhancing diffusion of colour images, Proc. VII National Symposium on Pattern Rec. and Image Analysis, Barcelona, 1 (1997) 239-244. 321

23. A. P. Witkin, "Scale space filtering", Proc. Int. Joint Conf. On Artificial Intelligence, pp. 1019-1023, 1983. 319 